\title{
Competencias geoespaciales para la mejora de la calidad de vida de las personas mayores: el proyecto OUTDOOR ICT
}

\author{
José Jesús Delgado Peña \\ Departamento de Geografía. Universidad de Málaga, España \\ jdelgado@uma.es
}

\begin{abstract}
There are numerous tools in the field of digital mapping and geospatial skills nowadays. ICT learning and the development of e-competences on these issues could facilitate both the integration and also a higher standard of living to the elderly. By using web 2.0 mapping applications, users can create their own maps and share them with other people, what can mean a step forward in the empowderment of the citizens. The main objective of the OUTDOOR ICT project was to provide opportunities for exploring ways of applying knowledge gained in the field of ICT to the promotion of active lifestyle by using GPS devices in cultural routes.

Two aspects that we consider to be essential for people over fifty in present-day society converge on this experience, namely digital competence stimulation and promotion of active methodologies for learning such as field work through an awareness of heritage and environmental issues, and a more active and healthier lifestyle. To that end, we have developed a learning activity in the area of geocaching by means of common mobile GPS (mobile phones or tablets) with free software (as Google Maps or Goggles), using Malaga and Antequera historic city-centres as operation fields. The research methodology of the results has been extensively based on questionnaires, and a very positive feedback has been obtained from the participants.
\end{abstract}

Keywords: geospatial skills, Web 2.0 mapping, geocaching, GPS devices, citizenship empowerment.

\section{Resumen}

Existen numerosas herramientas en el ámbito de la cartografía digital y las competencias geoespaciales en nuestros días. El aprendizaje de nuevas tecnologías y el desarrollo de competencias digitales sobre estas cuestiones podrían facilitar tanto la integración como la mejora de la calidad de vida de las personas mayores. Mediante el uso de la cartografía web 2.0, los usuarios pueden crear sus propios mapas y compartirlos con otras personas, lo que puede significar un paso adelante en el empoderamiento ciudadano.

El principal objetivo del proyecto OUTDOOR ICT fue la creación de oportunidades para explorar formas de aplicar conocimiento adquirido en el campo de las TIC para la promoción de un estilo de vida activo mediante el uso de dispositivos GPS en rutas culturales.

Dos aspectos que consideramos esenciales para las personas de más de 50 años en la sociedad actual convergen en esta experiencia: el estímulo de las competencias digitales y el uso de metodologías activas de aprendizaje como el trabajo de campo mediante la sensibilización sobre cuestiones patrimoniales y medioambientales y el estímulo de un modo de vida más activo y saludable. Para tal fin, se ha desarrollado una actividad de aprendizaje en el área del geocaching mediante el uso de dispositivos GPS comunes (smartphones y tablets) con software libre (como Google Maps o Goggles), utilizando los centros históricos de las ciudades de Málaga y Antequera como campos de operaciones. La metodología de investigación de los resultados se basó fundamentalmente en cuestionarios, y se obtuvo una respuesta muy positiva por parte de los participantes.

Palabras clave: competencias geoespaciales, cartografía 2.0, geocaching, dispositivos GPS, empoderamiento ciudadano. 


\section{Introducción}

La creciente importancia de la geolocalización en la sociedad actual refuerza el uso del GPS. Un gran número de personas ya lo utilizan, aunque aún queda el reto de su empleo en la enseñanza y aprendizaje de la educación de adultos, y más concretamente del adulto mayor. El trabajo de campo cobra una nueva dimensión con el uso del GPS, al integrar la información procedente de este en otras informaciones de índole geográfica, de modo que aporta nuevas formas de enseñanza en lo referente a las TIC en el ámbito de la geografía.

En los últimos años, se ha generalizado la visión de la tierra representada en tres dimensiones multirresolución, posibilitada por una serie de acontecimientos, como son la mejora de la potencia de los ordenadores y de los programas; la expansión y mejora de internet; la creación de globos virtuales, como por ejemplo, Google Earth; las infraestructuras de datos espaciales (IDE) en el marco de la directiva europea INSPIRE; el incremento de sensores remotos y terrestres, y más recientemente, las tecnologías emergentes que vienen de la mano de los dispositivos móviles (apps). Todo esto ha supuesto una revolución en la información geográfica, consistente en el incremento de la cantidad de geoinformación y la multiplicidad de formatos y posibilidades de acceso a esta (De Lázaro y Delgado, 2013).

A estas mejoras tecnológicas, hay que añadir la enorme proliferación de las aplicaciones y recursos existentes en la Web 2.0, que posibilita la creación colaborativa de contenidos de diferente naturaleza, destacando la cartografía digital, entre otros. Es, por tanto, necesario un esfuerzo para mejorar el aprendizaje de competencias geoespaciales en la población adulta mayor con el fin de aminorar los efectos de la brecha digital tanto como sea posible, así como ofrecerles conocimientos y destrezas en pos de una mejora de su calidad de vida.

Todo esto puede enmarcarse en la línea de la Agenda Digital 2020 de la Unión Europea, que pretende impulsar este campo científico-tecnológico, y en el programa recientemente creado por la ONU denominado «Global Geospatial Information Management» (UN-GGIM, 2014), que está relacionado con el manejo de la información geoespacial.

La utilidad del GPS se deriva del aprendizaje, comprensión y aprovechamiento de los datos toma- dos en el territorio que visitamos a partir de los dispositivos móviles y su integración en aplicaciones informáticas que nos permitan ver las imágenes de dicho lugar. Esto es hoy posible mediante las citadas WebSIG (Milson, 2012).

Vamos a exponer algunas sugerencias para su empleo, pensando especialmente en el adulto mayor y desde el enfoque ya señalado de la Web 2.0, en la cual la persona adopta un papel activo con respecto a los contenidos y aplicaciones existentes en internet, y puede participar activamente en la incorporación de nuevos contenidos, muchos de ellos con un significado o soporte geoespacial, y con diferentes propósitos.

El análisis territorial desde la perspectiva de divulgar realidades espaciales del entorno que habitamos es un aspecto de aplicación fundamental. Se deriva del trabajo de campo, siendo fundamental el uso del GPS para la obtención de datos y su georreferenciación. Algunos ejemplos de análisis territorial, en función de distintos objetivos, serían los siguientes (De Lázaro y Delgado, 2013):

- Detección de barreras para la movilidad. Para ello sería necesario rastrear el barrio y tomar con el GPS el punto exacto de aquellos lugares de difícil acceso para personas mayores o impedidas, bien porque no exista un paso de peatones, bien porque el pavimento esté deteriorado, bien porque los coches aparquen donde no deben, etc. Una vez recogidos los puntos, se pueden localizar en Google Earth o en ArcGIS Online y elaborar la cartografía pertinente. Podría aportar un valor añadido al estudio el enviarlo a las autoridades locales para que tomaran las medidas oportunas. La localización exacta de los puntos siempre ayudará a una resolución del problema más rápida.

- Detección de vertederos incontrolados. De manera similar al caso anterior, sería necesario rastrear el entorno y tomar con el GPS el punto exacto de aquellos lugares utilizados como vertederos incontrolados.

- Detección de puntos negros de contaminación acústica. Para ello sería necesario rastrear el barrio y tomar con el GPS el punto exacto de aquellos lugares conflictivos respecto a la contaminación acústica. En ese sentido, existen aplicaciones sencillas disponibles que nos pueden ayu- 
dar a medir los niveles de ruido del lugar donde nos encontremos en ese momento.

- Organización de una ruta o itinerario para presentar los principales monumentos o hitos de interés del lugar que habitamos o que nos gustaría destacar. Podemos acompañar estas rutas con imágenes y textos, y tener esta información completamente accesible desde internet.

- Búsqueda de aplicaciones o páginas web que nos permitan participar con informaciones georeferenciadas de interés. Un ejemplo de ello sería Google Goggles, aplicación que nos permite, haciendo una foto desde nuestro dispositivo móvil de un monumento u obra de arte, obtener otras imágenes de dicho elemento, así como información relativa a este. Es una herramienta de construcción social del conocimiento, por lo que cualquier usuario, además de consultar la información e imágenes existentes, puede añadir nuevas entradas.

En definitiva, el uso del GPS en combinación con las aplicaciones WebSIG fomenta una metodología activa, participativa, global e integradora, de modo que potencia el aprendizaje interdisciplinar y transdisciplinar y abarca el saber, saber hacer y saber ser, que el informe Delors (1996) formula. Favorece el análisis territorial y capacita al adulto mayor para comprender la multicausalidad de los procesos espaciales. Además, el fomento de las competencias digitales en esta población es crucial, pues la actual sociedad de la información, en un mundo globalizado, representa un verdadero reto que afrontar para las personas mayores. Esta sociedad es producto de la informatización generalizada de la información y de la revolución digital, y brinda un enorme abanico de posibilidades en lo que respecta a la información, la comunicación y la gestión de actividades en el día a día. Las personas mayores valoran el aprendizaje de aspectos relacionados con la informática desde una triple perspectiva: como un medio de comunicación con sus familiares y amigos, de manera que se reducen los efectos del aislamiento; como una vía de relación social, con lo que se mantienen informados en un entorno en continuo cambio, y como un recurso de ejercicio mental, de mantenerse activos (Delgado Peña et al., 2009: 133-136). La presente experiencia parte de todas estas premisas.

\section{EI proyecto OUTDOOR ICT}

\subsection{Objetivos y actividades}

El principal objetivo del proyecto OUTDOOR ICT (ref. 2011-1-HU1-GRU06-03650-2), proyecto financiado dentro del Programa de Aprendizaje Permanente de la Unión Europea, fue la creación de oportunidades para explorar formas de aplicación del conocimiento adquirido en el campo de las TIC para la promoción de un estilo de vida más activo y saludable. Para ello, hemos desarrollado una experiencia de aprendizaje en el ámbito del geocaching mediante el uso de dispositivos GPS móviles de uso frecuente (móviles o tablets) con software de uso libre (como Google Maps o Goggles), utilizando como terreno de actuación el casco histórico de las ciudades de Málaga y Antequera.

El geocaching se presenta como una actividad ampliamente extendida y cuya aplicación en el adulto mayor conlleva numerosos beneficios: mejora de las competencias espaciales y digitales, estímulo de la actividad física, sensibilización con respecto al patrimonio natural o cultural, etc. Es una actividad integradora de ejercicio físico, mental y dominio de la tecnología (aplicaciones web, telefonía móvil, etc.) en la que la edad no es un impedimento para su realización, ya que puede ser adaptada a todas las edades y niveles (Tejedor Lorenzo, 2006). Dicha actividad, que vio la luz por primera vez en mayo de 2000 , consiste, generalmente, en esconder objetos en el campo o en la ciudad, apuntar sus coordenadas y hacerlas públicas para que otras personas puedan efectuar su búsqueda mediante un dispositivo de localización GPS (Cameron, 2004; Dyer, 2004; Sherman, 2004; Gillin y Gillin, 2010). En este sentido, elegimos algunos monumentos clave y establecimos un itinerario de uno a otro siguiendo una lista de coordenadas que introducíamos en dichos dispositivos, de modo que la pantalla nos mostraba la ruta más adecuada.

En lugar de encontrar un «tesoro», algo físico concreto, el tesoro lo conformaba el propio monumento, y se establecían en cada una de las paradas dos pruebas: una de observación y otra de búsqueda de información en internet (figura 1). De esta forma, se estimulaba simultáneamente el desarrollo de competencias geográficas de vital importancia como son la orientación, la interpretación de la cartografía 
(plano de la ciudad) y la observación del entorno, junto a las competencias digitales al hacer uso de un software de geolocalización (Google Maps y Google Street View) en dispositivos cotidianos. Como la actividad se llevaba a cabo en equipos, también se estimulaba la interrelación y el desarrollo de competencias sociales. Hay que señalar, no obstante, que de manera previa a la salida de campo, se desarrolló una sesión en el aula de informática con el fin de

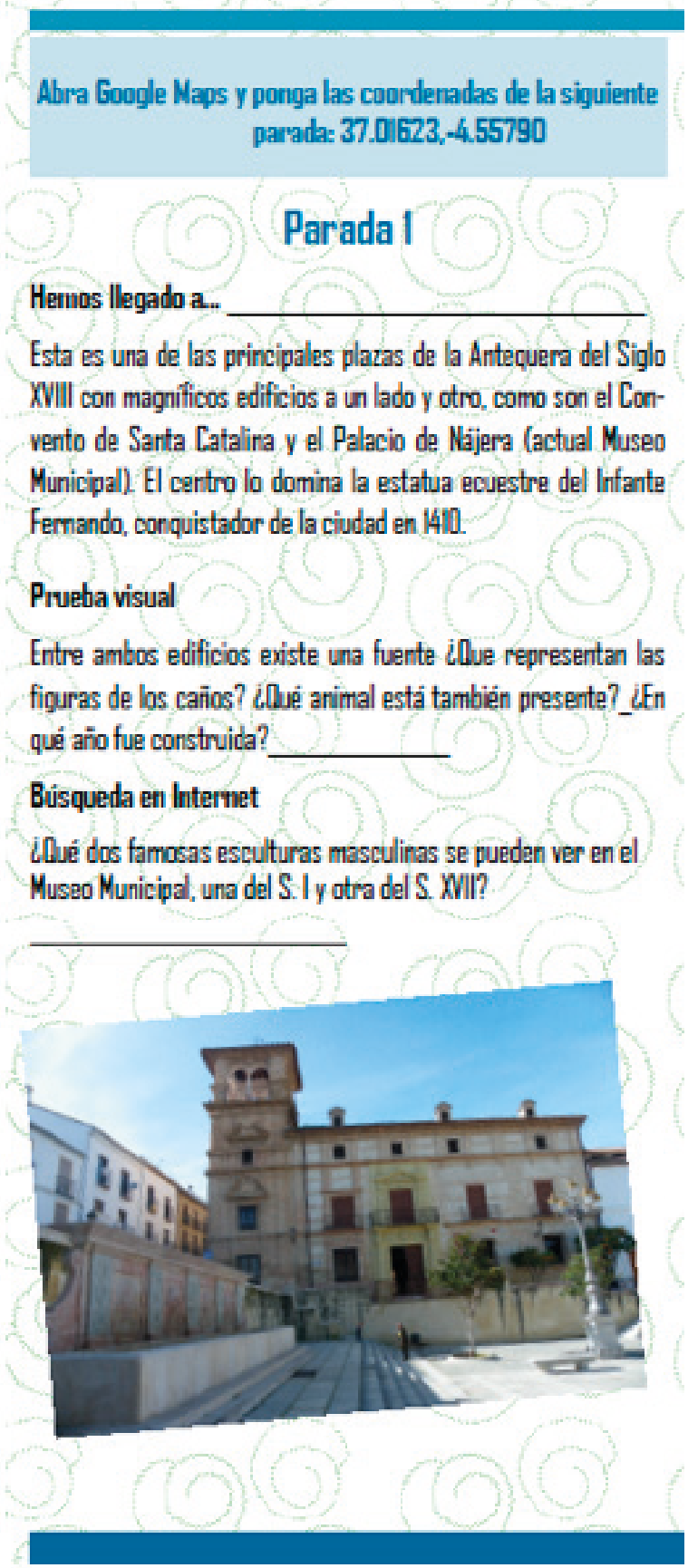

Figura 1. Actividades de la parada 1 de Antequera introducir al alumnado en todas estas aplicaciones informáticas, bastante desconocidas, en general, para el grupo objeto de dicha experiencia.

Habría que puntualizar una serie de aspectos importantes a la hora del diseño de una ruta en este ámbito (Delgado y Fernández, 2013): en primer lugar, es necesario establecer correctamente la longitud de esta, adaptándola al tiempo disponible y al número de paradas, no olvidando además que, debido a la naturaleza del público objetivo, no debe entrañar dificultades físicas excesivas. En el caso de Antequera, por ejemplo, hemos establecido una ruta que cubre unos 1950 metros en seis paradas. Puede verse la ruta en detalle mediante el enlace: $<$ http:// adventures.garmin.com/en-US/by/ruta-por-sevilla -este/geocaching-in-the-old-town-of-antequera/ \#overview>.

En segundo lugar, es crucial establecer un hilo conductor o argumento en el itinerario, algún aspecto histórico, ideológico o algún personaje que pueda aparecer en diferentes paradas de la ruta, de modo que se complete la información de manera progresiva. Para Antequera, el hecho histórico que enhebra nuestra ruta es la conquista de la ciudad mediante dos personajes fundamentales: el infante D. Fernando, el conquistador, y Santa Eufemia, una de las patronas, que aparecen de manera recurrente de alguna $\mathrm{u}$ otra forma en diversas paradas. Así, a lo largo del itinerario, se suceden datos complementarios, y se configura al final una historia coherente y perfectamente argumentada con las pruebas de observación y de búsqueda de internet de las diversas paradas. Desde el punto de vista patrimonial, este tipo de actividades de conocimiento y sensibilización puede

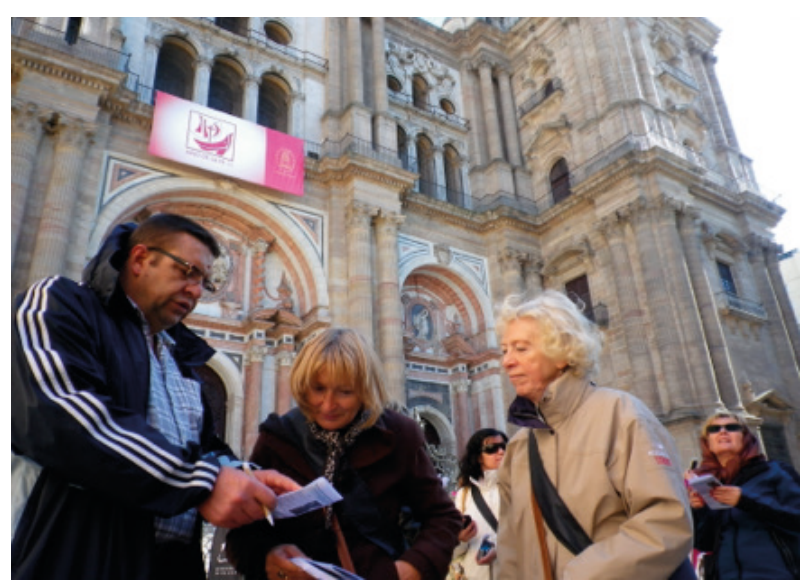

Figura 2. Grupo de participantes europeos en el itinerario de Málaga 
conllevar efectos muy positivos, más aún si hacemos uso de anécdotas o leyendas que enriquezcan el recorrido, y despierten el interés y la motivación de los participantes. Hay que subrayar la importancia del componente experiencial de lo aprendido, de tal modo que ligando los contenidos del itinerario a sentimientos de bienestar, de júbilo, al descubrir un detalle curioso o interesante, o al escuchar una anécdota divertida, por ejemplo, el proceso de aprendizaje pueda verse profundamente reafirmado.

\subsection{Resultados y conclusiones}

Para evaluar dicha actividad se llevó a cavo un estudio exploratorio mediante una metodología cuantitativa basada en cuestionarios de valoración completados por los participantes al final de la experiencia. Tras las dos actividades realizadas se obtuvieron 27 encuestas válidas completadas por alumnos/as del Aula de Mayores de la Universidad de Málaga y por participantes del proyecto pertenecientes a otras entidades socias. La muestra no es representativa de la población total, si bien sirve como estudio exploratorio previo (tabla 1). La elección de los participantes se basa en los criterios siguientes: $a$ ) edad a partir de 55 años (y hasta 84 años en nuestro caso); $b$ ) representación variada de las principales características (género, nivel educativo, nivel socio-económico, etc.), y c) como ya hemos señalado, relacionados de antemano con el proyecto.

Tabla 1. Ficha técnica de la investigación cuantitativa

\begin{tabular}{|l|l|}
\hline \multicolumn{1}{|c|}{ Ámbito de estudio } & \multicolumn{1}{c|}{$\begin{array}{c}\text { Aula de Mayores } \\
\text { (Universidad de Málaga) }\end{array}$} \\
\hline Metodología & $\begin{array}{l}\text { Cuestionario anónimo a } \\
\text { cumplimentar en papel }\end{array}$ \\
\hline $\begin{array}{l}\text { Población objeto de } \\
\text { estudio }\end{array}$ & Más de 100.000 habitantes \\
\hline $\begin{array}{l}\text { Tamaño de la } \\
\text { respuesta }\end{array}$ & 25 participantes \\
\hline Error muestral & $20 \%$ \\
\hline Nivel de confianza & $95 \% ; \mathrm{Z}=1,96 ; \mathrm{p}=\mathrm{q}=0,50$ \\
\hline
\end{tabular}

Fuente: J. García Mestanza.
En la encuesta valorativa efectuada, se valoraron una amplia variedad de aspectos, y se marcó una de las cinco posibilidades para las afirmaciones propuestas (Escala Likert, donde $1=$ completamente en desacuerdo; $2=$ desacuerdo; $3=$ ni de acuerdo ni en desacuerdo; 4 = de acuerdo; 5 = totalmente de acuerdo). Los resultados de las cuestiones planteadas se reflejan en las figura 3. Como se aprecia en esta gráfica resumen sobre los diferentes aspectos planteados en la cuarta pregunta referentes a la actividad formativa llevada a cabo, prácticamente todos los ítems analizados presentan las máximas puntuaciones (al menos el $96 \%$ de los participantes han contestado a los ítems con 4 y 5), señal de su satisfacción general, tanto por los aspectos aprendidos como por la metodología y recursos llevados a cabo.

Si hacemos una especial reflexión sobre aquellos ítems con una valoración inferior, vemos que no coinciden con los referentes al desarrollo de la actividad, a su satisfacción personal o a la metodología práctica utilizada, cuyos resultados son óptimos. Algo inferiores fueron los resultados sobre la posibilidad de uso y utilidad de lo aprendido de manera posterior al curso, lo que muestra la necesidad de reforzar la aplicabilidad de lo aprendido tras la actividad formativa, mediante, por ejemplo, la aplicación más patente de los dispositivos de geolocalización en el día a día, para encontrar, por ejemplo, alguna dirección necesaria, o utilizar aplicaciones Web 2.0 de interés, entre otras. Por los resultados vemos que se valora muy positivamente el trabajo en equipo y la posibilidad de compartir lo aprendido con otros. Finalmente, y como cabía de esperar, el trabajo de campo está mejor valorado que la sesión teórica en el aula. El componente experiencial y más íntimamente ligado con la realidad cercana aporta, sin duda alguna, un matiz de motivación difícil de emular en el aula.

En definitiva, la vivencia de los participantes, la planificación, el desarrollo de la actividad, así como sus futuras aplicaciones, muestran una excelente acogida por parte de los participantes.

La última cuestión está integrada por cuatro valoraciones que van desde el 0 (nada) al 10 (absolutamente) a las siguientes cuestiones: uso del GPS, realización de rutas GPS, utilización de programas para crear rutas y utilización de Google Maps u otros programas similares (figura 4). Aunque los resultados se muestran positivos, es patente la necesidad de profundizar en estos temas mediante actividades 
y metodologías que puedan ayudar al adulto mayor a conocer más y mejor las potencialidades del uso de las tecnologías referentes a la geolocalización (GPS, geocaching, aplicaciones WebSIG, etc.).

Tras el análisis de las principales valoraciones de las encuestas, algunas conclusiones generales podrían ser las siguientes:

- La mayoría de los participantes muestran gran interés en los temas relacionados con el uso de nuevas tecnologías aplicadas a aspectos geográficos como la orientación o la sensibilización hacia el entorno más cercano desde una perspectiva del análisis territorial.

- Se muestran, además, motivados para continuar participando en actividades de aprendizaje en esta dirección, preferiblemente mediante metodologías de enseñanza activa, como las salidas de campo, en nuestro caso.

- En relación con la metodología de enseñanza, se ha visto claramente la necesidad de vincular los conocimientos adquiridos con las experiencias y vivencias del adulto mayor, acercándoles las potencialidades de aplicación de lo aprendido a su rutina diaria y a favor de una mejora de la calidad de vida.

La experiencia llevada a cabo aporta información acerca de un tema de mucha actualidad y relevancia, como es el de la adquisición de competencias geográficas mediante la utilización de las TIC por parte del adulto mayor, que es consciente de que estas pueden favorecer el aprendizaje de competencias útiles e interesantes para su vida diaria. Prácticamente la totalidad afirman tener previsto continuar realizando actividades en esta dirección, por lo que sería necesario ofrecer un mayor número de programas, cursos o actividades que satisfagan dichas necesidades emergentes. Parece necesario desarrollar los servicios adecuados que exige esta demanda, cuyo porcentaje de población interesada es cada vez más elevado, tanto mediante la creación de cursos específicos y de las infraestructuras y equipamientos necesarios, como a través de la formación de personal especializado para este perfil de alumnado.

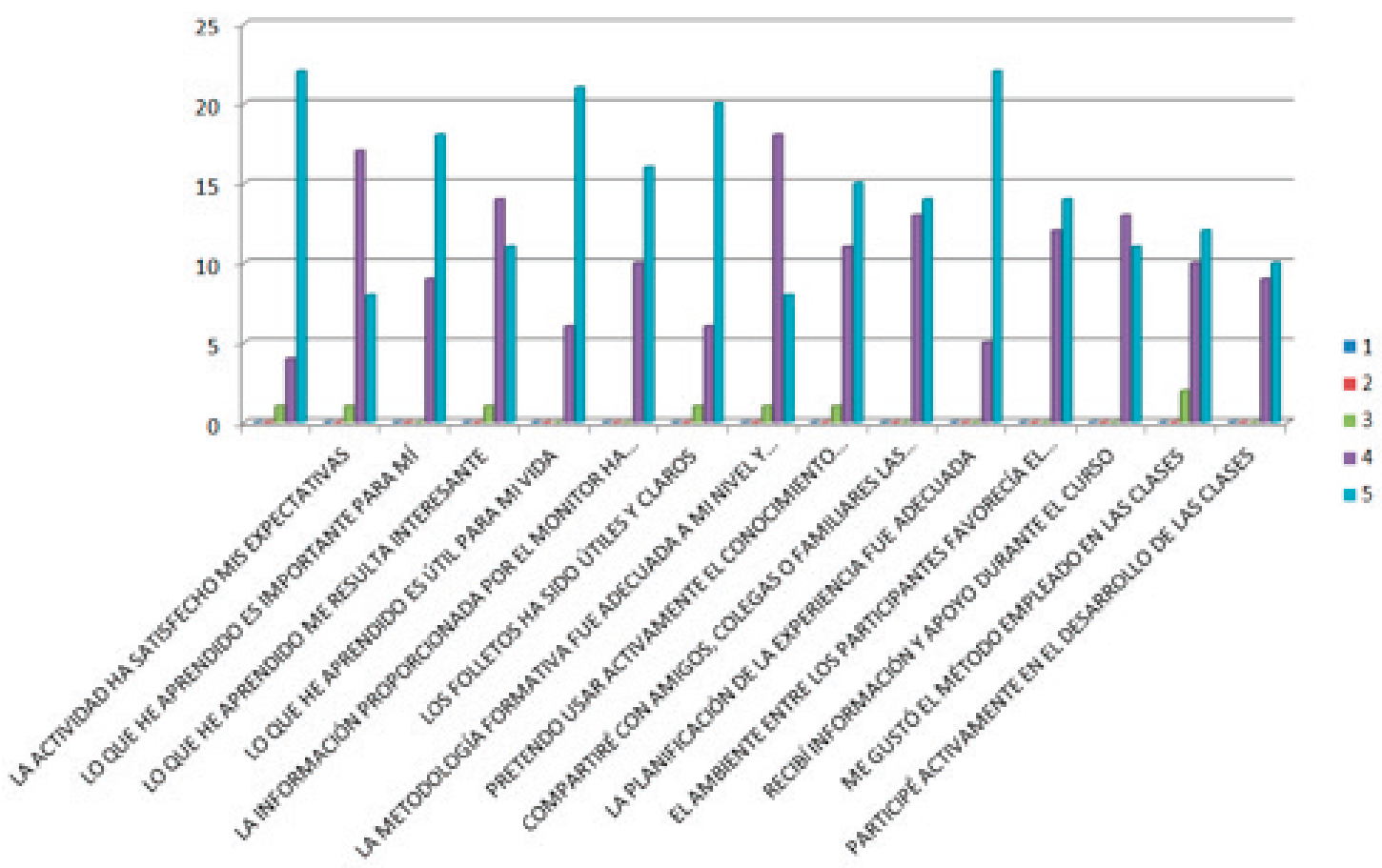

Figura 3. Respuestas de algunas de las cuestiones fundamentales del cuestionario de valoración 

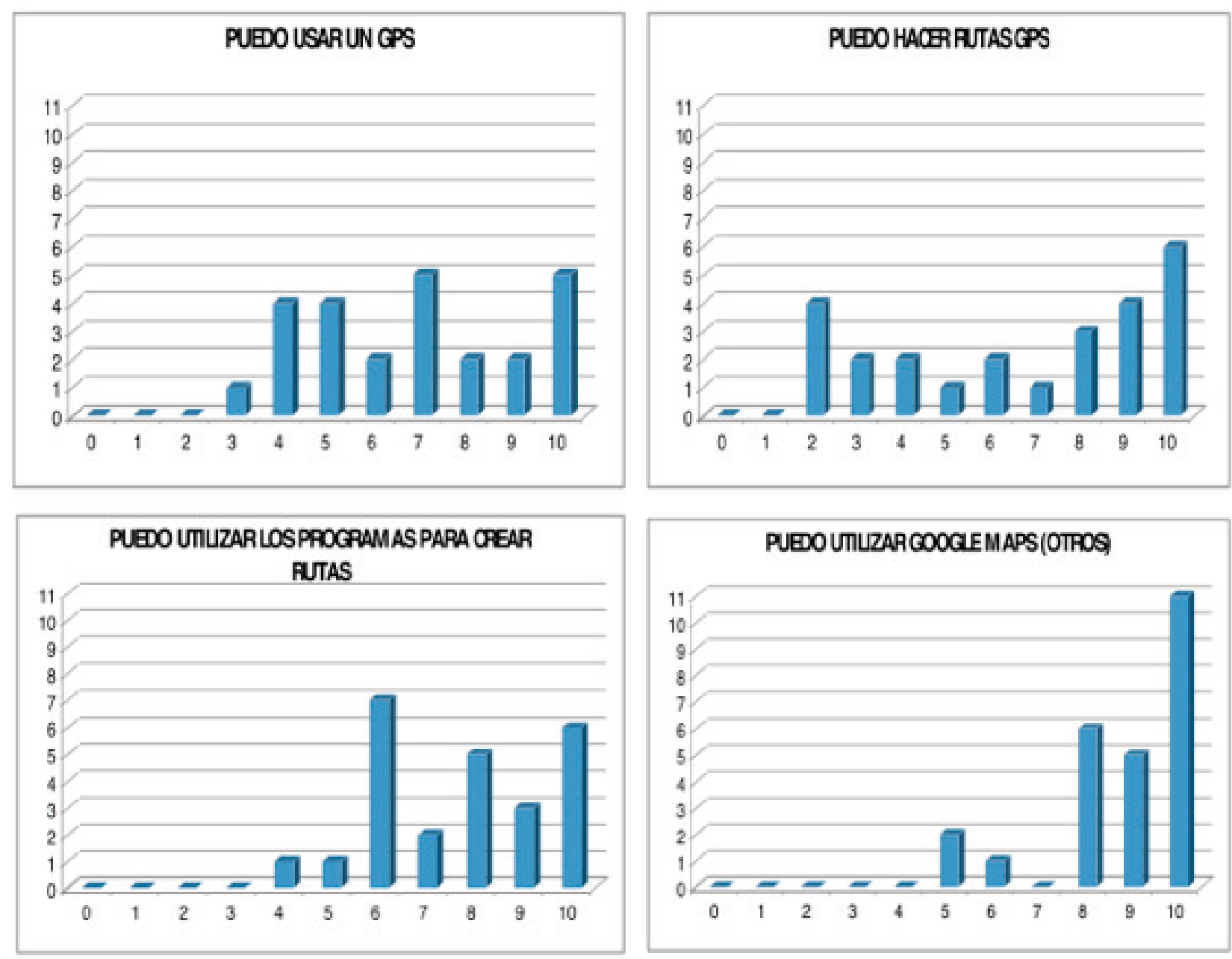

Figura 4. Distribución de la población encuestada con relación al uso del GPS, a la posibilidad de hacer rutas GPS, a la posibilidad de utilizar programas para crear rutas y al uso de Google Maps

\section{Referencias}

Cameron, L. (2004). The Geocaching Handbook (Falcon Guide): Guildford, EE. UU.: The Globe Pequot Press. De Lázaro y Torres, M. L. y Delgado Peña, J. J. (2013). Geolocation, a world of possibilities for people in later life. En J. J. Delgado (ed.), Geographic and geolocation competences for people in later life (pp. 18-25). Málaga, España: BPS Creatividad.

Delgado Peña, J. J. (2009). Competencias sociales y digitales en los Programas Universitarios para Mayores de la Universidad española (2008/0276). Consultado en la Base de datos del Programa de Estudios y Análisis de Ministerio de Educación de España. http://138.4.83.162/mec/ayudas/CasaVer.asp?P=29 359.

Delgado Peña, J. J. y Fernández González, J. C. (2013). Competencias digitales y geolocalización en la enseñanza del adulto mayor: ejemplo en el casco histórico de Antequera. En M. C. Moreno, M. M. Gallego y C. I. Gallego (eds.), Retos educativos de la cultura andaluza en una sociedad global (pp. 55-65). Málaga, España: Grupo de Investigación HUM-689. 
Delgado Peña, J. J. y García Mestanza, J. (2013). Competencias digitales geográficas y trabajo de campo en un ámbito urbano: el proyecto OUTDOOR ICT. En APG (ed.), A cidade, um laboratório para a educaçao geográfica (pp. 349-364). Oporto, Portugal: Universidade de Porto.

Delors, J. (1996). Los cuatro pilares de la Educación. En J. Delors (ed.), La educación encierra un tesoro. Informe a la UNESCO de la Comisión Internacional sobre la Educación para el siglo XXI (pp. 91-103). Madrid: Santillana/UNESCO.

Dyer, M. (2004). The essential Guide to Geocaching. Golden, EE. UU.: Fulcrum Publishing.

Gillin, P. y Gillin, D. (2010). The Joy of Geocaching. Chicago, EE. UU.: Linden Publishing

Google Goggles (n.d.). Consultado en: https://support.google.com/websearch/answer/166331.

Milson, A. J. (2012). SIG en la nube: WebSIG para la enseñanza de la Geografía. Didáctica Geográfica, 12, pp. 111-124.

Sherman, E. (2004). Geocaching. Hike and Seek with your GPS. New York, EE. UU.: Springer Verlag.

Tejedor Lorenzo, J. C. (2006). El GPS y sus aplicaciones en las actividades físicas en el medio natural en el ámbito escolar. Efdeportes.com Revista Digital, 97. Consultado en: http://www.efdeportes.com/efd97/gps.htm.

UN-GGIM. (2014). About UN-GGIM. Consultado en: http://ggim.un.org/default.html. 